\title{
SENSOR FAULT DETECTION BY SPARSITY OPTIMIZATION
}

\author{
Bingxuan Li, Hang Yu, Justin Dauwels, Kay Soon Low \\ School of Electrical and Electronic Engineering \\ Nanyang Technological University, Singapore
}

\begin{abstract}
Measurement faults in control systems may result in permanent damages to the system components. Therefore, sensor validation is essential before the measurements are used for any system reconfiguration. In this paper, a statistical approach for sensor fault identification is proposed. Specifically, the potential sensor fault is assumed to be an additive bias term in the measurement model. The problem of fault identification is formulated as a least-squares optimization problem with an $\ell_{1}$ penalty on the bias term. An algorithm is further introduced to determine the regularization parameter automatically. Experimental results show that the proposed method can accurately detect multiple sensor failures from noisy measurements.
\end{abstract}

Index Terms - sensor validation, bias detection, analytical redundancy, $\ell_{1}$ regularization selection

\section{INTRODUCTION}

Measurements in critical systems such as nuclear power plants [1] and aircrafts [2] should be acquired accurately, especially when the information is used for feedback. Sensor validation and its practical application in real-life systems has been investigated over the last three decades. The idea of sensor validation is to detect the sensor failure in the system based on multiple (possibly redundant) sensor measurements and the constraint relations (CRs) between them. Those two options are referred to as the hardware redundancy and analytical redundancy respectively. Obviously, installing multiple redundant sensors is helpful to identify and mitigate sensor faults, however, it increases the costs of the system.

Sensor validation is often performed locally for the sake of simplicity. Majority voting method and threshold check method are the most popular methods. Majority voting [3] employs sufficient hardware redundancy (more than 2 sensors) to validate a set of sensors. Although this method has the virtue that the validation results will not be affected by the uncertainties in the system, hardware redundancy of sensors may be costly in terms of space and weight budget. On the other hand, the threshold method validates sensors in a system by means of the nominal values, cf. [4]. However, many problems can arise from the choice of the threshold, such as false alarms caused by the system dynamic behavior. To solve this problem, a dynamic threshold method is proposed in [5, 6] using polynomial chaos observer, yet the sensor faults are not completely isolated from other physical faults in the system. Alternatively, soft computing methods such as fuzzy methods have also been applied to generate the dynamic threshold $[1,7,8,9,10]$. The resulting sensor values are selected based on the confidence level of each redundant sensor and their fuzzy membership parameters during the process of data fusion and threshold generation [9]. Unfortunately, the fuzzy algorithms are generally computationally expensive and the physical meaning of the parameter tuning is not obvious. Principle component analysis (PCA) is also employed to detect sensor faults [12]. A reduced order prediction is retrieved based on the correlation of the measurements. However, the method is only applicable under the assumption that the correlation between the faulty and the normal sensors is smaller than that between the normal sensors, which is incorrect in the case of constant sensor bias. Another approach is presented in [2] and [11], where the sensor relations are captured by a Bayesian network. The posteriori probability of the failure of a single sensor is then obtained through belief propagation, incorporating the measurements from the other sensors as well as commands to verify the selected sensor. Since the latter sensors may also be corrupted, the results obtained in this manner may not be accurate. Consequently, it is often necessary to consider fault diagnosis on a global system level instead of locally, and consider all sensors and CRs simultaneously. Along these lines, in [4] the CRs in a boiler thermal power plant are considered together, in order to validate all the sensors in the system without discrimination. The validity level of a sensor reflects the number of consistent CRs that are involved. However, the method involves complicated structural analysis and hypothesis testing. Another drawback is that parameter tuning by experts is required.

To take full advantage of the CRs in a system and to provide compatibility for different system structures, we formulate the problem of sensor fault detection as a least-squares optimization problem with an $\ell_{1}$ penalty for the sensor bias. CRs associated with hardware or analytical redundancy are imposed as constraints on the $\ell_{1}$ regularized least-squares optimization problem. A similar problem formulation is also adopted in [13]. However, the regularization parameter is fixed regardless of different failure scenarios and noise levels. Our experiments indicate that fixed regularization parameter 
will lead to biased estimates of faulty sensors. Instead, we propose to determine the regularization parameter automatically from the data based on a bootstrap approach [15]. With proper selection of the regularization parameter, the proposed method yields accurate sensor fault identification and also infers the sensor bias.

The paper is organized as follows. In Section 2, we present the proposed $\ell_{1}$ regularized least-squares optimization method for sensor fault detection. In Section 3 we explain how the regularization parameter can be selected in an automated manner. In Section 4 we consider two case studies, involving hardware and analytical redundancy. In Section 5 we offer concluding remarks.

\section{2. $\ell_{1}$ REGULARIZATION}

In this section, we first introduce the least-squares optimization with $\ell_{1}$ regularization, and subsequently, discuss its application to the sensor validation problem.

We denote by $\boldsymbol{c} \in \mathbb{R}^{N}$ and $\boldsymbol{b} \in \mathbb{R}^{N}$ the true state vector and bias vector respectively. We define the sensor measurement model as:

$$
y_{i}=c_{i}+b_{i}+\varepsilon_{i},
$$

where $c_{i}$ is the true value for the $i^{\text {th }}$ sensor, $y_{i}$ is the corresponding reading, $b_{i}$ is the sensor fault or bias, $i \in$ $\{1,2, \ldots, N\}$ denotes the sensor index, and $N$ is the total number of sensors. The measurement noise $\varepsilon_{i}$ is assumed to be Gaussian white noise with zero mean and variance $\sigma^{2}$. Note that both $c_{i}$ and $b_{i}$ are unknown.

Naively, one may try to infer $\boldsymbol{b}$ and $\boldsymbol{c}$ by solving the following least-squares problem:

$$
(\hat{\boldsymbol{b}}, \hat{\boldsymbol{c}})=\min _{\boldsymbol{b}, \boldsymbol{c}}\|\boldsymbol{y}-\boldsymbol{b}-\boldsymbol{c}\|^{2},
$$

where $\boldsymbol{y}$ is the measurement vector. Clearly this problem is ill-posed, as the bias $\boldsymbol{b}$ and the true sensor value $\boldsymbol{c}$ cannot be distinguished in (2).

To address this issue, we make the reasonable assumption that only a small fraction of the sensors are biased. Equivalently, the bias vector $\boldsymbol{b}$ is assumed to be sparse. Such prior knowledge can be integrated into the problem by introducing an $\ell_{1}$ regularization term into the objective function (1). Commonly $\ell_{1}$ regularization is applied in signal processing and statistics to induce sparsity. Furthermore, as mentioned in Section 1, constraint relations (CRs) $A c=0$ can also be leveraged to generate better estimates of the true sensor values and the sensor biases. Taken together, the regularized leastsquares optimization problem can be written as:

$$
(\hat{\boldsymbol{b}}, \hat{\boldsymbol{c}})=\min _{\boldsymbol{b}, \boldsymbol{c}}\|\boldsymbol{y}-\boldsymbol{b}-\boldsymbol{c}\|^{2}+k\|\boldsymbol{b}\|_{1} \text { s.t. } A \boldsymbol{c}=0 .
$$

Note that the matrix $A$ in Eq. (3) is constructed with the parameters of the CRs. For example, let the state vector $c$ be the voltage and the current $[V, I]$ across a resistor $R$. Then $A=[1,-R]$ according to Ohm's Law.

To reduce the number of unknowns in Eq. (3), an equivalent unconstrained problem can be formulated as:

$$
(\hat{\boldsymbol{b}}, \hat{\boldsymbol{a}})=\min _{\boldsymbol{b}, \boldsymbol{a}}\|\boldsymbol{y}-\boldsymbol{b}-Z \boldsymbol{a}\|^{2}+k\|\boldsymbol{b}\|_{1},
$$

where $Z$ contains basis vectors of the null space of $A$. The dimension of the vector $\boldsymbol{a}$ is $\operatorname{Nullity}(A)$. Although the $\ell_{1}$ penalty makes the estimates $\hat{\boldsymbol{b}}$ and $\hat{\boldsymbol{c}}$ different from the true bias and states, the estimates of the zero pattern of $\boldsymbol{b}$ is unbiased and consistent with the ground truth. The proposed method can therefore identify the faulty sensors reliably.

We can extend (4) to include $L$ samples of measurements $\boldsymbol{y}_{\ell}(\ell=1,2, \ldots, L)$ :

$$
(\hat{\boldsymbol{b}}, \hat{\boldsymbol{a}})=\min _{\boldsymbol{b}, \boldsymbol{a}} \frac{1}{L} \sum_{\ell=1}^{L}\left\|\boldsymbol{y}_{\ell}-\boldsymbol{b}-Z \boldsymbol{a}\right\|^{2}+k\|\boldsymbol{b}\|_{1} .
$$

The optimization problem (5) can be solved by the projected scaled sub-gradient (gafni-bertsekas variant) solver [16]. Note that the regularization parameter $k$ governs the trade-off between satisfying the CRs with the sensor readings and minimizing the number of biased sensors. By properly selecting $k$, we can correctly detect the biased sensors, which will be discussed in the next section.

\section{REGULARIZATION PARAMETER}

Our proposed method for error identification in sensors consists of the following steps:

1. Check whether there is inconsistency in the measurement vector with CRs. If $\|A(m) \boldsymbol{y}\|_{1}<\|A(m)\|_{1} \boldsymbol{b}_{\text {th }}$ for each row $m$ in matrix $A$, then the CR consistency is satisfied. $\boldsymbol{b}_{\text {th }}$ here represents the bias threshold vector. An empirical choice for bias threshold of each sensor is $3 \sigma$, where $\sigma$ is the sensor noise variance.

2. When inconsistency exists, the BINCO method [15] is applied for regularization selection. To obtain sufficient disturbance with a small sample size, bootstrap resampling is adopted, i.e. $L$ samples of measurement vectors are resampled with replacement to form $M$ data sets, and each set includes $L$ measurement vectors. For each of the $M$ data sets and each $k$ value in the given range, the optimization procedure in Eq. (5) is repeated to obtain the estimated bias and states. Specifically, the BINCO method is proceeded as follows:

(a) The selection frequency for each sensor can be obtained as $x_{i}=B_{i} / M$, where $B_{i}$ is the number of times $b_{i}>0$. The resulting $x$ generates an empirical probability density function $f^{k}$. A typical selection of regularization parameter $k$ will result in a U-shaped $f^{k}$ [15] as shown in Fig. 1.

(b) $f^{k}$ can be decomposed into the "normal" portion and the "bias" portion $f^{k}=(1-\pi) f_{0}^{k}+\pi f_{1}^{k}$, where $\pi$ is the ratio of biased sensors existing in the real system. $f_{0}^{k}$ is the density function of the normal sensors and $f_{1}^{k}$ is the density function of the biased ones. The minimum density point $V_{\min }$ is determined in the fitted curve of $f^{k}$ in Fig. 1. The functional form of the "normal" portion $\tilde{f}_{0}^{k}(x \mid \rho)$ can be estimated by fitting $f^{k}(x), x \in\left(0, V_{\min }\right]$ into a binomial distribution with a Beta prior on $\rho$ as in [15]. 




Fig. 1: Empirical density function of $x_{i}$.

(c) Extend $\tilde{f}_{0}^{k}$ to the region $x \in\left[V_{\min }, 1\right)$. For a given threshold of selection frequency $\gamma \in(0,1)$, FDR is defined as $\sum_{x \geq \gamma} \tilde{f}_{0}^{k}(x) / \sum_{x \geq \gamma} f^{k}(x)$.

(d) Choose the minimum $\gamma^{*}$ such that $\operatorname{FDR}\left(\gamma^{*}, k\right)<\alpha$, where $\alpha$ is a predefined threshold.

(e) The number of truly biased sensors is estimated as $N_{\mathrm{s}}(k)=\left(1-\operatorname{FDR}\left(\gamma^{*}, k\right)\right) \sum_{x \geq \gamma^{*}} f^{k}(x)$.

3 . Choose $k_{\text {opt }}$ that gives the maximum $N_{\text {bias }}$. Then the corresponding bias threshold is determined as

$T_{\mathrm{opt}}=\min \left(b_{i} \mid \forall i: x_{i}>\gamma^{*}\right)$.

4. Learn the sensor bias using constraint relations with healthy measurement for the detected faulty sensors.

\section{RESULTS AND DISCUSSION}

In this section, we assess the performance of the proposed method for two cases: hardware redundancy and analytical redundancy. We also investigate the stability of the proposed method when the nullity of $A$ is small.

\subsection{Hardware redundancy}

Here we consider the scenario where 100 (redundant) sensors measure the same quantity $c$ (thus $N=100$ ). Such situation is often referred to as "hardware redundancy". We generate $L$ samples $y_{\ell}(1)$. A certain fraction of the sensors are selected at random to be biased. Their bias $b$ is random uniformly distributed within the range $(0,10)$. The sensor noise variance $\sigma^{2}$ is set to 1 in this experiment. The BINCO method [15] is employed to determine the regularization parameter in the range $[0.1,1]$, and the FDR threshold $\alpha$ is set to $10^{-10}$. The mean square error (MSE) for estimating $\boldsymbol{b}$ is depicted in Fig. 2 for different sample size $L$.

As can be seen from Fig. 2, the MSE decreases as we increase the sample size $L$. Moreover, the MSE remains small even for a significant fraction of biased sensors (e.g., 30\%).

Next, we apply the proposed method for sensor validation and fault isolation in three cases with different noise levels $\left(\sigma^{2}=0.01,0.1,1\right) . L=10$ for each case. In the case $\sigma^{2}=$ 1 , we also show the results when $L=100$ for comparison. We average over 100 trials and compute the correct detection rate (CDR) as a function of biased sensor ratio. In Fig. 3, CDR $\geq 80 \%$ is achieved with a proper choice of $L$ when less than $50 \%$ of the sensors are biased, regardless of the noise level in the channel. Moreover, by comparing the results of $L=10$ and $L=100$, we observe that increasing the sample



Fig. 2: MSE of the proposed method for estimating $\boldsymbol{c}$ for varying number $L$ of samples.



Fig. 3: Correct detection rate (CDR) of the proposed method for three different noise levels.

size helps improve the estimation accuracy when the sensor readings are very noisy (i.e., $\sigma^{2}=1$ ).

\subsection{Analytical redundancy}

We now consider a static electric network, specifically, a resistive network. Suppose that the voltages and the currents at all resistors are measured by sensors. The relation between the $P$ CRs and the $N$ sensors is summarized in Table 1 [17].

Table 1: CRs for resistive network.

\begin{tabular}{|l|l|l|l|}
\hline Topology & $N$ & $P$ & No. of Resistors $N_{\mathrm{R}}$ \\
\hline Serial & $N_{\mathrm{R}}+2$ & $N_{\mathrm{R}}$ & $N_{\mathrm{R}} \geq 2$ \\
Parallel & $N_{\mathrm{R}}+3$ & $N_{\mathrm{R}}+1$ & $N_{\mathrm{R}} \geq 2$ \\
$\triangle$ & 9 & 6 & 3 \\
\hline
\end{tabular}

From Table 1, we can conclude that $\operatorname{Nullity}(A)=2$ for static electric networks with only parallel and series configurations, and $\operatorname{Nullity}(A)=3$ for the $\triangle$-configuration. We construct a simple parallel circuit, depicted in Fig. 4, to validate the proposed method. In this circuit, $R_{p}$ and $R_{s}$ represent the load and the wire loss respectively. The circuit can be adjusted to arbitrary scale by a specified odd number $N_{\mathrm{R}}$ of resistors. The CRs for the state variables $c$ are derived from the Kirchhoff's laws. Other parameters related to the following simulations in this subsection are listed in Table 2.

We first investigate how the noise variance $\left(\sigma^{2}=0.01,1\right)$ affects the identification of the biases. We randomly select sensors to be biased and set the bias value within the given range $|b|_{\max }$. The mean square error (MSE) between the estimated and true states averaged over 200 trials is shown in Fig. 5. In each trial, $k$ is specified by the BINCO method with FDR threshold $\alpha=10^{-10}$. Fig. 5 shows that the MSE is typically very small if less than $50 \%$ of the sensors are bi- 
Table 2: Circuit example: simulation parameters.



Fig. 4: Circuit example.

ased. We can also see that smaller noise variance yields more accurate estimates. Additionally, the MSE increases with the number of biased sensors for larger percentages (above 45\%) of biased sensors, as expected.

Next, we consider the effect of the penalty parameter $k$ on the estimates. Fig. 6 shows the CDR as a function of the biased sensor ratio, including the results of several fixed $k$ values and of the BINCO method. For the BINCO method, the FDR threshold is $\alpha=10^{-10}$. The noise variance $\sigma^{2}=1$ in this experiment. Let us first focus on the scenario when $k$ is fixed. When a small $k$ is used, the estimated $\hat{b}$ vector is dense. Consequently, the CDR is low due to the false negatives. On the other hand, a large $k$ results in a $\hat{b}$ that is more sparse than the true one, thus some biased sensors cannot be detected. In contrast, the BINCO method allows us to infer the optimum pair of $k_{\mathrm{opt}}$ and $T_{\mathrm{opt}}$ from the measurement vector $\boldsymbol{y}$ in an automated manner. As a result, the CDR resulting from the BINCO method is always higher than that resulting from all the choices of fixed $k$, as shown in Fig. 6. We further test the performance of the BINCO method for different values of the bias. We consider three random instances. In each case, the same bias $\boldsymbol{b}$ is assigned to 10 sensors selected at random in the circuit. We then change the value of $\boldsymbol{b}$ sequentially. The results averaged over 10 trials are summarized in Fig. 7. Obviously, the results generated by the BINCO method are robust to the location of the faulty sensors. Furthermore, we can find that the CDR is quite high (above 90\%) when the bias value is larger than 0.1 , and it grows with the given bias

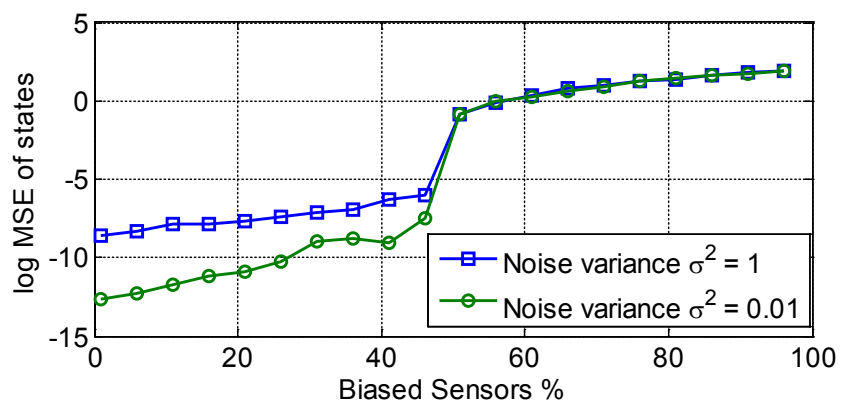

Fig. 5: MSE of state estimation.



Fig. 6: Correct Detection Rate for fixed $k$.

value, since large bias values make it easier to differentiate faults from noise. Interestingly, the values of $k_{\mathrm{opt}}$ determined by the BINCO method are in a small range, i.e., $[0.2,0.7]$, for different given bias values. By contrast, the threshold $T_{\mathrm{opt}}$ changes for different values of bias (see Fig. 8) substantially, implying that the BINCO method can automatically tune the parameters $k_{\mathrm{opt}}$ and $T_{\mathrm{opt}}$ in different cases.

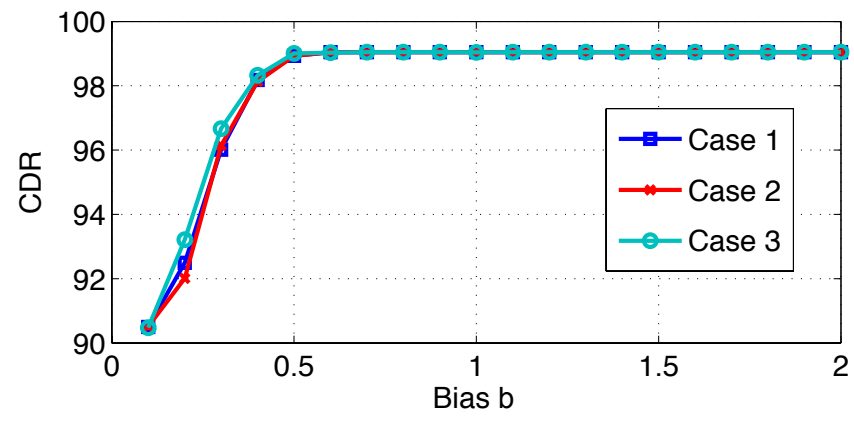

Fig. 7: Correct Detection Rate for different $\boldsymbol{b}$.

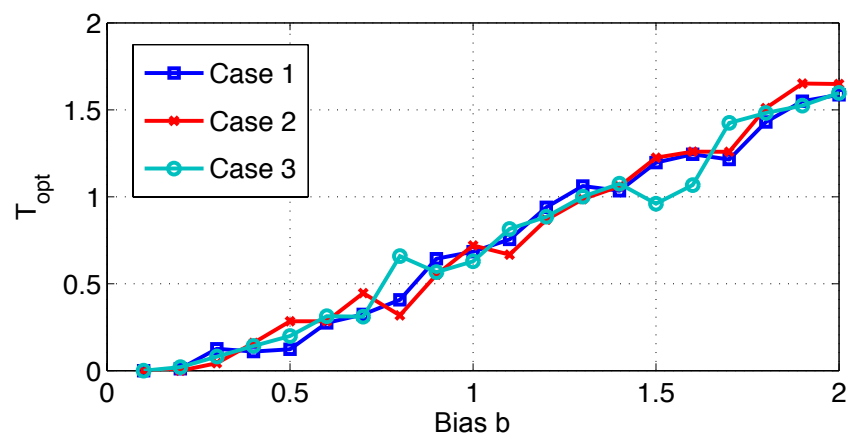

Fig. 8: Threshold $T_{\text {opt }}$ for different $\boldsymbol{b}$.

In summary, the BINCO method performs well at high noise level, yielding favorable CDRs; it also enables us to perform sensor bias identification without needing to tune parameters, which is beneficial in practical applications.

\section{CONCLUSIONS}

We proposed a novel method for sensor validation using least square optimization combined with $\ell_{1}$ regularization. The numerical results show that with automated tuning of the regularization parameter $k$, the sensor bias errors can be successfully identified, and the bias values can be accurately estimated. In future work, we will extend our approach to dynamical systems. 


\section{REFERENCES}

[1] Eryurek, E. and Upadhyaya, B.R., "Sensor validation for power plants using adaptive backpropagation neural network," IEEE Transactions on Nuclear Science, vol. 37, no. 2, pp. 1040-1047, 1990.

[2] Ole J. Mengshoel, Adnan Darwiche and Serdar Uckun, "Sensor Validation using Bayesian Networks," Proc. 9th International Symposium on Artificial Intelligence, Robotics, and Automation in Space (iSAIRAS), 2008; Available from: http://works.bepress.com/ole_mengshoel/22

[3] S.K. Yung and D.W. Clarke, "Local sensor validation," Measurentent and Control, vol. 22, no. 5, pp. 132-141, 1989.

[4] Lee, K.Y.; Chan-Eom Park, "Sensor value validation based on implicit sensor redundancy for reliable operation of power plants," IEEE Transactions on Energy Conversion, vol. 20, no. 2, pp. 373-380, 2005.

[5] Huimin Li, Monti, A., Ponci, F., Weilin Li, Min Luo and D'Antona, G., "Voltage Sensor Validation for Decentralized Power System Monitor Using Polynomial Chaos Theory," IEEE Transactions on Instrumentation and Measurement, vol. 60, no. 5, pp. 1633-1643, 2011.

[6] Huimin Li, Monti, A. and Ponci, F., "A Fuzzy-Based Sensor Validation Strategy for AC Motor Drives," IEEE Transactions on Industrial Informatics, vol. 8, no. 4, pp. 839-848, 2012.

[7] Goebel, K. and Weizhong Yan, "Correcting Sensor Drift and Intermittency Faults With Data Fusion and Automated Learning," IEEE Transactions on Systems Journal, vol. 2, no. 2, pp. 189-197, 2008.

[8] Frolik, J., M. Abdelrahman, and P. Kandasamy, "A confidence-based approach to the self-validation, fusion and reconstruction of quasi-redundant sensor data," IEEE Transactions on Instrumentation and Measurement, vol. 50, no. 6, pp. 1761-1769, 2001.

[9] Alag, S., A.M. Agogino, and M. Morjaria, "A methodology for intelligent sensor measurement, validation, fusion, and fault detection for equipment monitoring and diagnostics," Artif. Intell. Eng. Des. Anal. Manuf., vol. 15, no. 4 , pp. 307-320,2001.

[10] Iyengar, S.S. and Prasad, L., "A general computational framework for distributed sensing and fault-tolerant sensor integration," IEEE Transactions on Systems, Man and Cybernetics, vol. 25, no. 4, pp. 643-650, 1995.

[11] Ibarguengoytia, P.H., Sucar, L.E. and Vadera, S., "Real time intelligent sensor validation," IEEE Transactions on Power Systems, vol. 16, no. 4, pp. 770-775, 2001.
[12] Dunia, R., et al., "Identification of faulty sensors using principal component analysis." AIChE Journal, 1996. 42(10): p. 2797-2812

[13] D. Gorinevsky, S. Boyd, and S. Poll, "Estimation of Faults in DC Electrical Power System,"' Proceedings IEEE Conference on Decision and Control, pp. 43344339, 2009.

[14] Meinshausen, N. and P. Bühlmann, "Stability selection," Journal of the Royal Statistical Society, Series B (Statistical Methodology), vol. 72, no. 4, pp. 417-473, 2010.

[15] S. Li, L. Hsu, J. Peng and P. Wang, "Bootstrap Inference for Network Construction," Annals of Applied Statistics,vol. 7, no. 1, pp. 391-417, 2011.

[16] Mark Schmidt, 2006 03/04/2012]; Available from: http://www.di.ens.fr/ mschmidt/Software/L1General.html

[17] J. B. Dennis. "Mathematical Programming and Electrical Networks," The Technology Press of The Massachusetts Institute of Technology, Cambridge, Mass., 1959; Available from: http://hdl.handle.net/1721.1/13366 